\title{
Regards
}

\section{L'interdisciplinarité en action : un chercheur engagé, Jean-Marie Legay}

Jean-Marie Legay, qui fut avec Marcel Jollivet et Gérard Mégie l'un des trois rédacteurs en chef « fondateurs " de Natures Sciences Sociétés, a joué un rôle primordial tout au long de la vie de la revue. Son action a été décisive, de la maturation progressive du concept éditorial par une équipe pluridisciplinaire au lancement de NSS en 1993, à la mise en œuvre méthodique du projet intellectuel, le faisant ainsi progresser, et à sa diffusion au-delà de la communauté initiale. Il a complété cette action en créant en 2004 la collection "Indisciplines » co-éditée par l'association NSS-Dialogues et les éditions Quæ.

Son décès, le 8 avril 2012, a été durement ressenti, en particulier au sein de la communauté NSS qu'il avait contribué à former, structurer et développer. Sa disparition a d'autant plus affecté certains d'entre nous, que son action significative dans la revue n'était qu'une facette de ses activités et de ses engagements. Au travers de projets de recherche communs, de directions de thèses, mais aussi de rencontres informelles, nous avons pris la mesure de l'intelligence de ce chercheur, à la fois exigeant, rigoureux et subtilement indiscipliné.

C'est pourquoi, nous avons sollicité des chercheurs, des enseignants et des étudiants qui l'avaient côtoyé de près. Leurs contributions, réparties sur deux numéros de la revue (celui-ci et le prochain ${ }^{1}$ ), témoignent, dans des styles divers, au-delà des nombreux échanges scientifiques et des liens d'amitié, de la diversité de ses apports dans la science et dans la société et de leur cohérence, aussi bien dans son rôle de chercheur et d'enseignant que dans celui de passeur de frontières.

Claude Millier et la Rédaction

\footnotetext{
Auteur correspondant : claude.millier@agroparistech.fr

1 Cf., dans ce numéro, les textes de Marcel Jollivet, JeanDominique Lebreton, Franck Varenne, Nigel G. Yoccoz, Richard Tomassone, Francis Laloë. Dans le numéro suivant, seront publiés ceux d'Anne-Françoise Schmid, Nicole Mathieu, Sylvie Lardon, Dominique Pontier et Alain Pavé.
}

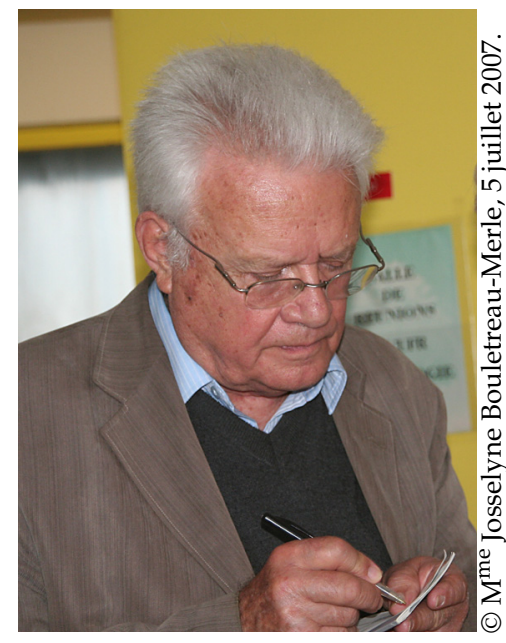

\section{Éléments de biographie ${ }^{2}$}

Jean-Marie Legay est né le 9 août 1925. Il a donc 20 ans le jour de Nagasaki, et il faut certainement y voir une des racines de son engagement politique.

Ingénieur agronome, spécialiste de la physiologie du ver à soie, il commence sa carrière à l'Inra à Saint-Christollès-Alès. Il quitte l'Inra pour l'Université de Lyon (devenue depuis Université Claude Bernard Lyon 1) à la fin des années 1950. Il y fonde un «laboratoire de biométrie », actuel Laboratoire de biométrie et biologie évolutive (UMR CNRS 5558), immergé chez les biologistes, qui explorera de multiples facettes de l'emploi des mathématiques en biologie. Il soulignait d'ailleurs souvent que la pluridisciplinarité entre mathématiques et biologie imposait « d'accepter les énoncés de la biologie». JeanMarie Legay est aussi un enseignant hors du commun, sachant coupler, là comme ailleurs, exigence conceptuelle et pragmatisme.

Il prend dans les années 1970 la responsabilité d'un groupe de travail " Méthodologie écologique » attaché au comité « Gestion des ressources naturelles

2 Rédaction: Jean-Dominique Lebreton. 
renouvelables » de la Délégation générale à la recherche scientifique et technique (DGRST). Ce modeste comité rassemble tant de talents qu'il devient rapidement un lieu privilégié de réflexion pluridisciplinaire, de la biologie vers les mathématiques et la modélisation, tout d'abord, puis, rapidement vers les sciences de l'homme et de la société.

Il a été notamment à la fin des années 1980 directeur adjoint du département des sciences de la vie au CNRS.

\section{Bibliographie indicative}

\section{Sur la modélisation}

Legay, J.-M., 1968. Éléments d'une théorie générale de la croissance d'une population, Bulletin of Mathematical Biophysics, 30, 33-46.

Legay, J.-M., 1971. Contribution à l'étude de la forme des plantes : discussion d'un modèle de ramification, Bulletin of Mathematical Biophysics, 33, 3, 387-401.

Legay, J.-M., 1973. La méthode des modèles, état actuel de la méthode expérimentale, Informatique et Biosphère, 1, 5-73.

Legay, J.-M., 1973. Introduction à l'étude des modèles à compartiments, Informatique et Biosphère, 119-145.

Legay, J.-M., Tomassone, R. (Eds), 1977. Biométrie et Écologie, Paris, Société française de biométrie.

Legay, J.-M., 1980. Bio-informatique, Encyclopædia Universalis, $1,288-291$

Legay, J.-M., 1984. Sur les relations biométrie-écologie, Bull. Ecol., 15, 117-119.

Legay, J.-M., 1987. Contribution à l'étude de la complexité dans les systèmes biologiques, in Actes du colloque de Solignac de 1985, Paris, CNRS Éditions, 147-165.

Legay, J.-M., 1986. Qu'est-ce que la biométrie ? Courrier du CNRS, 64, 56-61.

Legay, J.-M., 1986. Méthodes et modèles dans l'étude des systèmes complexes, Cahiers de la recherche-développement, $11,1-6$
Legay, J.-M., 1993. Une expérience est-elle possible ? in Lebreton, J.-D., Asselain, B. (Eds), Biométrie et Environnement, Paris, Masson.

Legay, J.-M., 1997. L'Expérience et le Modèle. Un discours sur la méthode, Paris, Inra Éditions.

Legay, J.-M., Tomassone, R., 1999. La comparaison de régressions orthogonales, Revue de Statistique Appliquée, XLVII, 2, 81-101.

\section{Sur l'interdisciplinarité}

Legay, J.-M., 1986. Quelques réflexions à propos d'écologie : défense de l'indisciplinarité, Acta CEcologica, CEcologia Generalis, 7, 4, 391-398.

Godard, O., Legay, J.-M., 1992. Entre disciplines et réalités, l'artifice des systèmes, in Jollivet, M. (Ed.), Sciences de la nature, sciences de la société. Les passeurs de frontières, Paris, CNRS Éditions, 243-257.

Legay, J.-M., 2002. Du ver à soie à la modélisation. Comment devient-on indiscipliné? (propos recueillis par A.-F.Schmid), Natures Sciences Sociétés, 10, 1, 59-63.

Legay, J.-M., Schmid, A.-F., 2004. Philosophie de l'interdisciplinarité. Correspondance (1999-2004) sur la recherche scientifique, la modélisation et les objets complexes, Paris, Pétra.

Jollivet, M., Legay, J.-M., 2005. Canevas pour une réflexion sur une interdisciplinarité entre sciences de la nature et sciences sociales, Natures Sciences Sociétés, 13, 184-188.

Legay, J.-M. (Ed.), 2006. L'Interdisciplinarité dans les sciences de la vie, Versailles, Quæ.

\section{Sur d'autres questions}

Legay, J.-M., 1972. Pourquoi deux sexes? Bull. Math. Biophysics, $34,2,297-304$.

Legay, J.-M., 1981. Qui a peur de la science? Travailleurs scientifiques, politique et société, Paris, Éditions sociales.

Legay, J.-M., 2004. À propos du livre de Chahdortt Djavann: Bas les voiles! Natures Sciences Sociétés, 12, 208-209. 